\title{
Compositional abstraction for interconnected systems over Riemannian manifolds: A small-gain approach
}

\author{
Asad Ullah Awan and Majid Zamani
}

\begin{abstract}
In this work, we propose a compositional approach for the construction of approximations for interconnected systems evolving on Riemannian manifolds. This allows for larger classes of systems than the ones considered in existing works defined only over Euclidean spaces. In the proposed framework, the approximation, itself a control system (possibly with a lower dimension), can be used as a substitute of the original system in the controller design process. We employ a notion of so-called simulation function, constructed using a (pseudo) Riemannian metric defined over the tangent bundle of the state space, to quantify the error between concrete interconnected control systems and their approximations. We provide a small-gain type condition that enables the construction of an abstraction for the interconnected control system compositionally.
\end{abstract}

\section{INTRODUCTION}

Controller synthesis for large-scale interconnected systems to achieve some complex specifications in a reliable and cost effective way is a formidable task. One line of research which has been explored to surmount this challenge is to use a simpler (e.g. lower dimension) (in)finite approximation (referred to as abstraction) of the given system as a substitute in the controller design process. Instead of synthesizing a controller to enforce the complex specifications over the output of the original system directly, one can synthesize a controller to enforce that specification on the output of the abstraction, and then refine that controller to the one for the original system. The original complex system and abstraction are related such that the error between their output behaviors can be quantified.

Instead of constructing abstractions of the complex system as a whole (monolithic approach), one can leverage the fact that many large-scale complex systems can be regarded as interconnected systems consisting of smaller control subsystems. This motivates a compositional approach for the construction of the abstractions in which abstractions of the original interconnected systems can be provided by constructing abstractions of the subsystems and their interconnections. Recently, there have been several results on

This work was supported in part by the European Union's Horizon 2020 Research and Innovation program under grant agreement no. 674875 and by the German Research Foundation (DFG) through the grant ZA 873/1-1. (c) 2018 IEEE. Personal use of this material is permitted. Permission from IEEE must be obtained for all other uses, in any current or future media, including reprinting/republishing this material for advertising or promotional purposes, creating new collective works, for resale or redistribution to servers or lists, or reuse of any copyrighted component of this work in other works.

A. U. Awan and M. Zamani are with the Department of Electrical and Computer Engineering, Technical University of Munich, D-80290 Munich, Germany. Email: $\{$ asad.awan, zamani\}@tum.de. the compositional construction of (in)finite abstractions of deterministic control systems including [1], [2], [3], and of a class of stochastic hybrid systems [4], [5].

All the aformentioned results in the context of (in)finite abstractions consider systems evolving over the Euclidean spaces. The state-space of many systems are Riemannian manifolds [6], and therefore, their analysis requires tools from differential geometry [7]. In this work, we propose techniques for compositional construction of infinite abstractions for interconnected control systems evolving over smooth Riemannian manifolds. We introduce a notion of so-called simulation functions constructed from (pseudo) Riemannian metric defined over the Cartesian product of the tangent bundle of the interconnected control system and that of its abstraction. Given a network of control subsystems and the simulation functions between them and their abstractions, we derive sufficient conditions based on small-gain type reasoning [8], guaranteeing that a network of abstractions quantitatively approximates the original network of concrete subsystems.

\section{CONTROL SyStemS}

\section{A. Notation}

The sets of non-negative integer and real numbers are denoted by $\mathbb{N}$ and $\mathbb{R}$, respectively. Those symbols are subscripted to restrict them in the usual way, e.g. $\mathbb{R}_{>0}$ denotes the positive real numbers. The symbol $\mathbb{R}^{n \times m}$ denotes the vector space of real matrices with $n$ rows and $m$ columns. For $a, b \in \mathbb{R}$ with $a \leq b$, the closed interval in $\mathbb{R}$ is denoted by $[a, b]$. For $a, b \in \mathbb{N}$ and $a \leq b$, we use $[a ; b]$ to denote the corresponding interval in $\mathbb{N}$. Given $N \in \mathbb{N}_{\geq 1}$, vectors $x_{i} \in \mathbb{R}^{n_{i}}, n_{i} \in \mathbb{N}_{\geq 1}$ and $i \in[1 ; N]$, we use $x=\left[x_{1} ; \ldots ; x_{N}\right]$ to denote the concatenated vector in $\mathbb{R}^{n}$ with $n=\sum_{i=1}^{N} n_{i}$. Given a vector $x \in \mathbb{R}^{n}$, we denote by $\|x\|$ the Euclidean norm of $x$. Given matrices $M_{1}, \ldots, M_{n}$, the notation $\operatorname{diag}\left(M_{1}, \ldots, M_{n}\right)$ represents a block diagonal matrix with diagonal matrix entries $M_{1}, \ldots, M_{n}$. Given a function $f: \mathbb{R}_{\geq 0} \rightarrow \mathbb{R}^{n}$, the (essential) supremum of $f$ is denoted by $\|f\|_{\infty}:=(\operatorname{ess}) \sup \{\|f(t)\|, t \geq 0\}$. A continuous function $\gamma: \mathbb{R}_{\geq 0} \rightarrow \mathbb{R}_{\geq 0}$, is said to belong to class $\mathcal{K}$ if it is strictly increasing and $\gamma(0)=0 ; \gamma$ is said to belong to $\mathcal{K}_{\infty}$ if $\gamma \in \mathcal{K}$ and $\gamma(r) \rightarrow \infty$ as $r \rightarrow \infty$. A continuous function $\beta: \mathbb{R}_{\geq 0} \times \mathbb{R}_{\geq 0} \rightarrow \mathbb{R}_{\geq 0}$ is said to belong to class $\mathcal{K} \mathcal{L}$ if, for each fixed $t$, the map $\beta(r, t)$ belongs to class $\mathcal{K}$ with respect to $r$, and for each fixed non zero $r$, the map $\beta(r, t)$ is decreasing with respect to $t$ and $\beta(r, t) \rightarrow 0$ as $t \rightarrow \infty$. An ( $n$-dimensional) manifold $\mathcal{M}_{n}$ is a pair $\left(\mathcal{M}_{n}, \mathcal{A}^{+}\right)$where $\mathcal{M}_{n}$ is a set and $\mathcal{A}^{+}$is a maximal atlas 
into $\mathbb{R}^{n}$, such that the topology induced by $\mathcal{A}^{+}$is Hausdorff and second countable. We denote the tangent space of $\mathcal{M}_{n}$ at $x \in \mathcal{M}_{n}$ by $\mathcal{T}_{x} \mathcal{M}_{n}$, and the tangent bundle of $\mathcal{M}_{n}$ by $\mathcal{T} \mathcal{M}_{n}=\bigcup_{x \in \mathcal{M}_{n}}\{x\} \times \mathcal{T}_{x} \mathcal{M}_{n}$. A curve on the manifold is a mapping $\gamma: I \subset \mathbb{R} \rightarrow \mathcal{M}_{n}$. A distance (or metric) $d: \mathcal{M}_{n} \times \mathcal{M}_{n} \rightarrow \mathbb{R}_{\geq 0}$ on a manifold $\mathcal{M}_{n}$ is a continuous positive function that satisfies $d(x, y)=0$ if and only if $x=y$ for each $x, y \in \mathcal{M}_{n}$, and $d(x, z) \leq d(x, y)+d(y, z)$ for each $x, y, z \in \mathcal{M}_{n}$. A (pseudo) Riemannian metric [9] on a smooth manifold $\mathcal{M}_{n}$ is a smoothly varying inner product on the tangent bundle $\mathcal{T} \mathcal{M}_{n}$ of manifold $\mathcal{M}_{n}$. Given $\mathcal{M}_{n}$, and a matrix valued map $G: \mathcal{M}_{n} \rightarrow \mathbb{R}^{n \times n}$ such that $G(x)$ is a positive (semi) definite matrix for each $x \in \mathcal{M}_{n}$, the (pseudo) Riemannian metric corresponding to the (pseudo) Riemannian structure $G$ is given by $\delta x^{T} G(x) \delta y$ for each $x \in$ $\mathcal{M}_{n}, \delta x \in \mathcal{T}_{x} \mathcal{M}_{n}$ and $\delta y \in \mathcal{T}_{x} \mathcal{M}_{n}$. Given two points $x, y \in$ $\mathcal{M}_{n}$, a smooth curve $\gamma:[0,1] \rightarrow \mathcal{M}_{n}$ such that $\gamma(0)=x$, and $\gamma(1)=y$, and a (pseudo) Riemannian structure $G$ defined on $\mathcal{M}_{n}$, we define the (pseudo) Riemannian energy functional as $E_{G}(\gamma)=\int_{0}^{1} \frac{\partial \gamma}{\partial s}^{T}(s) G(\gamma(s)) \frac{\partial \gamma}{\partial s}(s) d s$. For two points $z_{1}, z_{2} \in \mathcal{M}_{n}, \Gamma\left(z_{1}, z_{2}\right)$ denotes the set of piecewise continuous curves connecting $z_{1}$ and $z_{2}: \Gamma\left(z_{1}, z_{2}\right)=\{\gamma$ : $[0,1] \rightarrow \mathcal{M}_{n} \mid \gamma$ is piecewise continuous , $\gamma(0)=z_{1}, \gamma(1)=$ $\left.z_{2}\right\}$. Given two points $x, y \in \mathcal{M}_{n}$, a Riemannian structure $G$ defined on $\mathcal{M}_{n}, \underset{\gamma \in \Gamma(x, y)}{\arg \min } \int_{0}^{1} \sqrt{\frac{\partial \gamma}{\partial s}^{T}(s) G(\gamma(s)) \frac{\partial \gamma}{\partial s}(s)} d s$ is called a geodesic curve between $x$ and $y$ with respect to $G$.

\section{B. Control Systems}

Now, we define the class of control systems investigated in this paper.

Definition 2.1: The class of control systems studied in this paper is a tuple $\Sigma=\left(\mathcal{M}_{n}, \mathbb{R}^{m}, \mathbb{R}^{p}, \mathcal{U}, \mathcal{W}, f, \mathbb{R}^{q}, h\right)$, where

- $\mathcal{M}_{n}$ is an $n$-dimensional state manifold containing the origin, while $\mathbb{R}^{m}, \mathbb{R}^{p}$, and $\mathbb{R}^{q}$, are the external input, internal input, and output (Euclidean) spaces of dimension $m, p$, and $q$ respectively;

- $\mathcal{U}$ and $\mathcal{W}$ are subsets of sets of all measurable functions of time taking values in $\mathbb{R}^{m}$ and $\mathbb{R}^{p}$, respectively;

- $f: \mathcal{M}_{n} \times \mathbb{R}^{p} \times \mathbb{R}^{m} \rightarrow \mathcal{M}_{n}$ is the continuously differentiable state evolution map. We assume that $f(0,0,0)=0$;

- $h: \mathcal{M}_{n} \rightarrow \mathbb{R}^{q}$ is the continuously differentiable output map;

A control system $\Sigma$ satisfies

$$
\Sigma:\left\{\begin{array}{l}
\dot{\xi}(t)=f(\xi(t), v(t), \omega(t)) \\
\zeta(t)=h(\xi(t))
\end{array}\right.
$$

for any $v \in \mathcal{U}$ and any $\omega \in \mathcal{W}$, where a locally absolutely continuous curve $\xi: \mathbb{R}_{>0} \rightarrow \mathcal{M}_{n}$ is called a state trajectory of $\Sigma, \zeta: \mathbb{R}_{\geq 0} \rightarrow \mathbb{R}^{q}$ is called an output trajectory of $\Sigma$. We also write $\xi_{a v \omega}(t)$ to denote the value of the state trajectory at time $t \in \mathbb{R}_{\geq 0}$ under the input trajectories $v$ and $\omega$ from initial condition $\xi_{a v \omega}(0)=a$, where $a \in \mathcal{M}_{n}$. We denote by $\zeta_{a v w}$ the output trajectories corresponding to the state trajectory $\xi_{a v \omega}$.

\section{Definition 2.2: Given any}

$$
\Sigma=\left(\mathcal{M}_{n}, \mathbb{R}^{m}, \mathbb{R}^{p}, \mathcal{U}, \mathcal{W}, f, \mathbb{R}^{q}, h\right),
$$

the variational control system of $\Sigma$ is given by the tuple

$$
\delta \Sigma=\left(\mathcal{T} \mathcal{M}_{n}, \mathbb{R}^{m}, \mathbb{R}^{p}, \mathcal{U}, \mathcal{W}, \delta f, \mathbb{R}^{q}, \delta h\right),
$$

where for every $[x ; \delta x] \in \mathcal{T} \mathcal{M}_{n}, u \in \mathbb{R}^{m}, \delta u \in \mathbb{R}^{m}, w \in$ $\mathbb{R}^{p}$, and $\delta w \in \mathbb{R}^{p}$ :

$$
\begin{aligned}
\delta f(x, \delta x, u, \delta u, w, \delta w):= & \frac{\partial f}{\partial x}(x, u, w) \delta x+\frac{\partial f}{\partial u}(x, u, w) \delta u \\
& +\frac{\partial f}{\partial w}(x, u, w) \delta w \\
\delta h(x, \delta x):= & \frac{\partial h}{\partial x}(x) \delta x .
\end{aligned}
$$

Remark 2.3: If the control system $\Sigma$ does not have internal inputs, the definition of the control system in Definition 2.1 reduces to the tuple

$$
\Sigma=\left(\mathcal{M}_{n}, \mathbb{R}^{m}, \mathcal{U}, f, \mathbb{R}^{q}, h\right) .
$$

Correspondingly, the equation (II.1) describing the state and output trajectories reduces to:

$$
\Sigma:\left\{\begin{array}{l}
\dot{\xi}(t)=f(\xi(t), v(t)), \\
\zeta(t)=h(\xi(t)) .
\end{array}\right.
$$

We use the notion of control system in (II.2) later to refer to an overall interconnected control system. The variational control system of $\Sigma$ can be defined similar to Definition 2.2.

Definition 2.4: Let

$$
\Sigma=\left(\mathcal{M}_{n}, \mathbb{R}^{m}, \mathbb{R}^{p}, \mathcal{U}, \mathcal{W}, f, \mathbb{R}^{q}, h\right),
$$

and

$$
\hat{\Sigma}=\left(\mathcal{M}_{\hat{n}}, \mathbb{R}^{\hat{m}}, \mathbb{R}^{p}, \hat{\mathcal{U}}, \hat{\mathcal{W}}, \hat{f}, \mathbb{R}^{q}, \hat{h}\right),
$$

be two control subsystems with the same internal input and output space dimension. We define the augmented system

$$
\tilde{\Sigma}=\left(\mathcal{M}_{\tilde{n}}, \mathbb{R}^{\tilde{m}}, \mathbb{R}^{2 p}, \tilde{\mathcal{U}}, \tilde{\mathcal{W}}, \tilde{f}, \mathbb{R}^{q}, \tilde{h}\right),
$$

where $\mathcal{M}_{\tilde{n}}=\mathcal{M}_{n} \times \mathcal{M}_{\hat{n}}, \tilde{\mathcal{U}}=\mathcal{U} \times \hat{\mathcal{U}}, \tilde{\mathcal{W}}=\mathcal{W} \times \hat{\mathcal{W}}, \tilde{m}=$ $m+\hat{m}$, and for each $x \in \mathcal{M}_{n}, \hat{x} \in \mathcal{M}_{\hat{n}}, u \in \mathbb{R}^{m}, \hat{u} \in \mathbb{R}^{\hat{m}}$, $w \in \mathbb{R}^{p}$, and $\hat{w} \in \mathbb{R}^{p}$

$$
\begin{aligned}
\tilde{f}(\tilde{x}, \tilde{u}, \tilde{w}) & :=\left[\begin{array}{l}
f(x, u, w) \\
\hat{f}(\hat{x}, \hat{u}, \hat{w})
\end{array}\right], \\
\tilde{h}(\tilde{x}) & :=h(x)-\hat{h}(\hat{x}),
\end{aligned}
$$

where $\tilde{x}=[x ; \hat{x}], \tilde{u}=[u ; \hat{u}]$, and $\tilde{w}=[w ; \hat{w}]$.

Definition 2.5: Let $\Sigma=\left(\mathcal{M}_{n}, \mathbb{R}^{m}, \mathcal{U}, f, \mathbb{R}^{q}, h\right)$ and $\hat{\Sigma}=$ $\left(\mathcal{M}_{\hat{n}}, \mathbb{R}^{\hat{m}}, \hat{\mathcal{U}}, \hat{f}, \mathbb{R}^{q}, \hat{h}\right)$ be two control systems with the same output space dimension. We define the augmented system $\tilde{\Sigma}=\left(\mathcal{M}_{\tilde{n}}, \mathbb{R}^{\tilde{m}}, \tilde{\mathcal{U}}, \tilde{f}, \mathbb{R}^{q}, \tilde{h}\right)$, where $\mathcal{M}_{\tilde{n}}=\mathcal{M}_{n} \times \mathcal{M}_{\hat{n}}, \tilde{\mathcal{U}}=$ $\mathcal{U} \times \hat{\mathcal{U}}, \tilde{m}=m+\hat{m}$, and for each $x \in \mathcal{M}_{n}, \hat{x} \in \mathcal{M}_{\hat{n}}$, $u \in \mathbb{R}^{m}$, and $\hat{u} \in \mathbb{R}^{\hat{m}}$ :

$$
\begin{aligned}
\tilde{f}(\tilde{x}, \tilde{u}) & :=\left[\begin{array}{l}
f(x, u) \\
\hat{f}(\hat{x}, \hat{u})
\end{array}\right], \\
\tilde{h}(\tilde{x}) & :=h(x)-\hat{h}(\hat{x}),
\end{aligned}
$$

where $\tilde{x}=[x ; \hat{x}]$, and $\tilde{u}=[u ; \hat{u}]$. 


\section{Simulation Functions}

In this section, we introduce a notion of so-called simulation functions, which is used to quantify the closeness of output trajectories of the concrete systems and the ones of their abstractions.

Definition 3.1: Consider two control subsystems

$$
\Sigma=\left(\mathcal{M}_{n}, \mathbb{R}^{m}, \mathbb{R}^{p}, \mathcal{U}, \mathcal{W}, f, \mathbb{R}^{q}, h\right)
$$

and

$$
\hat{\Sigma}=\left(\mathcal{M}_{\hat{n}}, \mathbb{R}^{\hat{m}}, \mathbb{R}^{p}, \hat{\mathcal{U}}, \hat{\mathcal{W}}, \hat{f}, \mathbb{R}^{q}, \hat{h}\right)
$$

and the corresponding augmented system

$$
\tilde{\Sigma}=\left(\mathcal{M}_{\tilde{n}}, \mathbb{R}^{\tilde{m}}, \mathbb{R}^{2 p}, \tilde{\mathcal{U}}, \tilde{\mathcal{W}}, \tilde{f}, \mathbb{R}^{q}, \tilde{h}\right)
$$

as in Definition 2.4. Let

$$
\delta \tilde{\Sigma}=\left(\mathcal{T} \mathcal{M}_{\tilde{n}}, \mathbb{R}^{\tilde{m}}, \mathbb{R}^{2 p}, \tilde{\mathcal{U}}, \tilde{\mathcal{W}}, \delta \tilde{f}, \mathbb{R}^{q}, \delta \tilde{h}\right)
$$

be the variational control system of $\tilde{\Sigma}$ as defined in Definition 2.2. Suppose there exists some positive constants $\alpha$ and $\lambda$, a matrix valued function $G: \mathcal{M}_{\tilde{n}} \rightarrow \mathbb{R}^{\tilde{n} \times \tilde{n}}$ such that $G(\tilde{x})$ is a positive (semi) definite matrix for all $\tilde{x} \in \mathcal{M}_{\tilde{n}}$, functions $\psi_{\text {ext }} \in \mathcal{K}_{\infty} \cup\{0\}$, $\psi_{\text {int }} \in \mathcal{K}_{\infty} \cup\{0\}$ and a continuously differentiable function ${ }^{1} k: \mathcal{M}_{\tilde{n}} \times \mathbb{R}^{\hat{m}} \times \mathbb{R}^{p} \rightarrow$ $\mathbb{R}^{m}$ which satisfies $k(0,0,0)=0$, such that the following two conditions hold: ${ }^{2}$

- For any $\tilde{x} \in \mathcal{M}_{\tilde{n}}$ :

$$
G(\tilde{x}) \succeq \alpha\left(\frac{\partial \tilde{h}}{\partial \tilde{x}}\right)^{T}\left(\frac{\partial \tilde{h}}{\partial \tilde{x}}\right)
$$

- For any $[\tilde{x} ; \delta \tilde{x}] \in \mathcal{T} \mathcal{M}_{\tilde{n}}, \hat{u} \in \mathbb{R}^{\hat{m}}, \delta \hat{u} \in \mathbb{R}^{\hat{m}}$, $\hat{w} \in \mathbb{R}^{p}, \delta \hat{w} \in \mathbb{R}^{p}$, if we choose $u$ using the map $u=k(\tilde{x}, \hat{u}, \hat{w})$, then for all $w \in \mathbb{R}^{p}, \delta w \in \mathbb{R}^{p}$ :

$$
\begin{aligned}
& \delta \tilde{x}^{T}\left(\frac{\partial \tilde{f}^{T}}{\partial \tilde{x}} G(\tilde{x})+G(\tilde{x}) \frac{\partial \tilde{f}}{\partial \tilde{x}}+\frac{\partial G}{\partial \tilde{x}} \tilde{f}(\tilde{x}, \tilde{u}, \tilde{w})\right) \delta \tilde{x} \\
& +2 \delta \tilde{w}^{T} \frac{\partial \tilde{f}^{T}}{\partial \tilde{w}} G(\tilde{x}) \delta \tilde{x}+2 \delta \tilde{u}^{T} \frac{\partial \tilde{f}^{T}}{\partial \tilde{u}} G(\tilde{x}) \delta \tilde{x} \\
& \leq-\lambda \delta \tilde{x}^{T} G(\tilde{x}) \delta \tilde{x}+\psi_{\text {int }}(\|\delta w-\delta \hat{w}\|)+\psi_{\text {ext }}(\|\delta \hat{u}\|),
\end{aligned}
$$

where, $\delta \tilde{u}=[\delta u ; \delta \hat{u}], \delta u=\frac{\partial k}{\partial \tilde{x}} \delta \tilde{x}+\frac{\partial k}{\partial \hat{u}} \delta \hat{u}+\frac{\partial k}{\partial \hat{w}} \delta \hat{w}$, $\tilde{w}=[w ; \hat{w}]$, and $\delta \tilde{w}=[\delta w ; \delta \hat{w}]$.

then

$$
V_{G}(\tilde{x})=\inf _{\tilde{\gamma} \in \Gamma(\tilde{x}, 0)} \int_{0}^{1} \tilde{\gamma}^{\prime}(s)^{T} G(\tilde{\gamma}(s)) \tilde{\gamma}^{\prime}(s) d s,
$$

is called a simulation function from $\hat{\Sigma}$ to $\Sigma$ with respect to the (pseudo) Riemannian structure $G$. We call $\hat{\Sigma}$ (preferably with $\hat{n}<n)$ an abstraction of $\Sigma$ if there exists a simulation function from $\hat{\Sigma}$ to $\Sigma$.

The next theorem shows the usefulness of the existence of a simulation function in quantifying the closeness of two control subsystems.

\footnotetext{
${ }^{1}$ We refer to $k$ as the interface map.

${ }^{2}$ Here, for brevity, we do not write the arguments of the partial derivatives explicitly.
}

Theorem 3.2: Let $\Sigma=\left(\mathcal{M}_{n}, \mathbb{R}^{m}, \mathbb{R}^{p}, \mathcal{U}, \mathcal{W}, f, \mathbb{R}^{q}, h\right)$ and $\hat{\Sigma}=\left(\mathcal{M}_{\hat{n}}, \mathbb{R}^{\hat{m}}, \mathbb{R}^{p}, \hat{\mathcal{U}}, \hat{\mathcal{W}}, \hat{f}, \mathbb{R}^{q}, \hat{h}\right)$ be two control systems. Suppose $V_{G}$, associated with the (pseudo) Riemannian structure $G$ and the interface map $k$, is a simulation function from $\hat{\Sigma}$ to $\Sigma$, then there exists $\beta \in \mathcal{K} \mathcal{L}, \bar{\psi}_{\text {ext }} \in \mathcal{K}_{\infty} \cup\{0\}$, $\bar{\psi}_{\text {int }} \in \mathcal{K}_{\infty} \cup\{0\}$ such that for any $x \in \mathbb{R}^{n}, \hat{x} \in \mathbb{R}^{\hat{n}}, \hat{v} \in \hat{\mathcal{U}}$ and $\hat{\omega} \in \hat{\mathcal{W}}$, if we choose $v \in \mathcal{U}$ using $k$, then the following inequality holds for any $t \in \mathbb{R}_{\geq 0}$ and any $\omega \in \mathcal{W}$ :

$$
\begin{aligned}
\left\|\zeta_{x v \omega}(t)-\hat{\zeta}_{\hat{x} \hat{v} \hat{\omega}}(t)\right\| \leq & \beta\left(V_{G}(x, \hat{x}), t\right)+\bar{\psi}_{\text {ext }}\left(\|\hat{v}\|_{\infty}\right) \\
& +\bar{\psi}_{\text {int }}\left(\|\omega-\hat{\omega}\|_{\infty}\right) .
\end{aligned}
$$

Proof: Consider two points $\tilde{x}=[x ; \hat{x}] \in \mathcal{M}_{\tilde{n}}$ and $0 \in \mathcal{M}_{\tilde{n}}$, and a geodesic $\chi:[0,1] \rightarrow \mathbb{R}^{\tilde{n}}$, with respect to the (pseudo) Riemannian structure $G$, such that $\chi(0)=0$, and $\chi(1)=\tilde{x}$. The energy functional corresponding to this geodesic is given by

$$
V_{G}(\tilde{x})=E_{G}(\tilde{x}, 0)=\int_{0}^{1} \chi^{\prime}(s)^{T} G(\chi(s)) \chi^{\prime}(s) d s .
$$

Let $\tilde{\xi}_{\tilde{x} \tilde{\nu} \tilde{\omega}}=\left[\xi_{x \nu \omega} ; \hat{\xi}_{\hat{x} \hat{\nu} \hat{\omega}}\right]$ be the solution trajectory of $\tilde{\Sigma}$ for any initial condition $\tilde{x} \in \mathcal{M}_{\tilde{n}}$, under the external input trajectory $\tilde{\nu}=[\nu ; \hat{\nu}]$, where $\nu(t)=k\left(\tilde{\xi}_{\tilde{x} \tilde{\nu} \tilde{\omega}}(t), \hat{\nu}(t), \hat{\omega}(t)\right)$, for all $t \in \mathbb{R}_{\geq 0}$, for any $\hat{\nu} \in \hat{\mathcal{U}}$, and under internal input trajectory $\tilde{\omega}=[\omega ; \hat{\omega}]$, where $\omega \in \mathcal{W}$, and $\hat{\omega} \in \mathcal{W}$.

For a fixed $t \in \mathbb{R}_{\geq 0}$, consider the straight line $\hat{\eta}(s, t)$ $=s \hat{\nu}(t)$ in $s$, where $s \in[0,1]$. For any fixed $t \in \mathbb{R}_{\geq 0}$, the curve $\hat{\eta}(\cdot, t):[0,1] \rightarrow \mathbb{R}^{\hat{m}}$ is a geodesic, with respect to the Euclidean metric, on $\mathbb{R}^{\hat{m}}$ joining $\hat{\eta}(0, t)=0$ and $\hat{\eta}(1, t)=\hat{\nu}(t)$. For a fixed $t \in \mathbb{R}_{>0}$, consider the straight line $\tilde{\eta}_{w}(s, t)=\left[\begin{array}{l}\eta_{w}(s, t) \\ \hat{\eta}_{w}(s, t)\end{array}\right]=s\left[\begin{array}{c}\omega(t) \\ \hat{\omega}(t)\end{array}\right]$ in $s$, where $s \in[0,1]$. For any fixed $t \in \mathbb{R}_{\geq 0}$, the curve $\tilde{\eta}_{w}(\cdot, t):[0,1] \rightarrow \mathbb{R}^{2 p}$ is a geodesic, with respect to the Euclidean metric, on $\mathbb{R}^{2 p}$ joining $\tilde{\eta}_{w}(0, t)=0$ and $\tilde{\eta}_{w}(1, t)=\left[\begin{array}{l}\omega(t) \\ \hat{\omega}(t)\end{array}\right]$.

For any $s \in[0,1]$, let $\tilde{\phi}(s, \cdot): \mathbb{R}_{\geq 0} \rightarrow \mathbb{R}^{\tilde{n}}$ be the solution trajectory of $\tilde{\Sigma}$ from initial condition $\chi(s)$ under the external input $\tilde{\eta}_{u}(s, \cdot)$, where $\tilde{\eta}_{u}(s, t)=$ $\left[\begin{array}{c}k\left(\tilde{\phi}(s, t), \hat{\eta}(s, t), \hat{\eta}_{w}(s, t)\right) \\ \hat{\eta}(s, t)\end{array}\right]$, and the internal input $\tilde{\eta}_{w}(s, \cdot)$, where $\tilde{\eta}_{w}(s, t)=s\left[\begin{array}{l}\omega(t) \\ \hat{\omega}(t)\end{array}\right] \forall t \in \mathbb{R}_{\geq 0}$. Note that $\tilde{\phi}(0, t)=0$, and $\tilde{\phi}(1, t)=\tilde{\xi}_{\tilde{x} \tilde{\nu} \tilde{\omega}}(t)$.

For brevity, we denote $\frac{\partial}{\partial s} \tilde{\phi}(s, t)=: \tilde{\rho}(s, t)$. Note that

$$
\begin{aligned}
& \frac{\partial}{\partial t} \tilde{\rho}(s, t)=\frac{\partial^{2}}{\partial t \partial s} \tilde{\phi}(s, t)=\frac{\partial^{2}}{\partial s \partial t} \tilde{\phi}(s, t) \\
& =\frac{\partial}{\partial s} \tilde{f}\left(\tilde{\phi}(s, t), \tilde{\eta}_{u}(s, t), \tilde{\eta}_{w}(s, t)\right) \\
& =\frac{\partial \tilde{f}}{\partial \tilde{x}} \frac{\partial}{\partial s} \tilde{\phi}(s, t)+\frac{\partial \tilde{f}}{\partial \tilde{u}} \frac{\partial}{\partial s} \tilde{\eta}_{u}(s, t)+\frac{\partial \tilde{f}}{\partial \tilde{w}} \frac{\partial}{\partial s} \tilde{\eta}_{w}(s, t) \\
& =\frac{\partial \tilde{f}}{\partial \tilde{x}} \tilde{\rho}(s, t)+\frac{\partial \tilde{f}}{\partial \tilde{u}}\left[\begin{array}{c}
\left.\frac{\partial k}{\partial \tilde{x}} \tilde{\rho}(s, t)+\frac{\partial k}{\partial \hat{u}} \hat{\nu}(t)+\frac{\partial k}{\partial \hat{w}} \hat{\omega}(t)\right] \\
\hat{\nu}(t)
\end{array}\right] \\
& \quad+\frac{\partial \tilde{f}}{\partial \tilde{w}}\left[\begin{array}{c}
\omega(t) \\
\hat{\omega}(t)
\end{array}\right] .
\end{aligned}
$$


Define

$$
l(t)=\int_{0}^{1} \tilde{\rho}(s, t)^{T} G(\phi(s, t)) \tilde{\rho}(s, t) d s
$$

i.e. $l(t)$ is the energy functional of the curve $\tilde{\phi}(\cdot, t)$, with respect to $G$. We have

$$
\begin{aligned}
& \frac{d}{d t} l(t)=\int_{0}^{1} \frac{\partial}{\partial t} \tilde{\rho}(s, t)^{T} G(\tilde{\phi}(s, t)) \tilde{\rho}(s, t) d s \\
& =\int_{0}^{1} \tilde{\rho}^{T}\left(\frac{\partial \tilde{f}}{\partial \tilde{x}} G+G \frac{\partial \tilde{f}}{\partial \tilde{x}}+\frac{\partial G}{\partial \tilde{x}} f\right) \tilde{\rho} d s \\
& +2 \int_{0}^{1}\left[\frac{\partial k}{\partial \tilde{x}} \tilde{\rho}+\frac{\partial k}{\partial \hat{u}} \hat{\nu}+\frac{\partial k}{\partial \hat{w}} \hat{\omega}\right]^{T} \frac{\partial \tilde{f}^{T}}{\partial \tilde{u}} G \tilde{\rho} d s \\
& +2 \int_{0}^{1}\left[\begin{array}{c}
\omega \\
\hat{\omega}
\end{array}\right]^{T} \frac{\partial \tilde{f}^{T}}{\partial \tilde{w}} G \tilde{\rho} d s
\end{aligned}
$$

where, again, we dropped explicit arguments for clarity in the last expression. From (III.2), one has:

$$
\begin{aligned}
\frac{d}{d t} l(t) \leq & -\lambda \int_{0}^{1} \tilde{\rho}(s, t)^{T} G(\tilde{\phi}(s, t)) \tilde{\rho}(s, t) d s \\
& +\int_{0}^{1} \psi_{\operatorname{ext}}\left(\left\|\frac{\partial \hat{\eta}(s, t)}{\partial s}\right\|\right) d s \\
& +\int_{0}^{1} \psi_{\operatorname{int}}\left(\left\|\frac{\partial}{\partial s} \eta_{w}(s, t)-\frac{\partial}{\partial s} \hat{\eta}_{w}(s, t)\right\|\right) d s \\
\leq & -\lambda \int_{0}^{1} \tilde{\rho}(s, t)^{T} G(\tilde{\phi}(s, t)) \tilde{\rho}(s, t) d s \\
& +\psi_{\operatorname{int}}(\|\omega(t)-\hat{\omega}(t)\|) \int_{0}^{1} d s \\
& +\psi_{\operatorname{ext}}(\|\hat{\nu}(t)\|) \int_{0}^{1} d s \\
\leq & -\lambda l(t)+\psi_{\text {ext }}\left(\|\hat{\nu}\|_{\infty}\right)+\psi_{\text {int }}\left(\|\omega-\hat{\omega}\|_{\infty}\right) .
\end{aligned}
$$

It follows from the comparison lemma [10] that

$$
l(t) \leq \mathrm{e}^{-\lambda t} l(0)+\frac{1}{\lambda} \psi_{\text {ext }}\left(\|\hat{\nu}\|_{\infty}\right)+\frac{1}{\lambda} \psi_{\text {int }}\left(\|\omega-\hat{\omega}\|_{\infty}\right) .
$$

Note that $l(0)=V_{G}\left(\xi_{x \nu \omega}(0), \hat{\xi}_{\hat{x} \hat{\nu} \hat{\omega}}(0)\right)=V_{G}(\tilde{x})$. Now using the fact that for any $t \in \mathbb{R}_{\geq 0}, l(t)$ is not necessarily the minimum energy functional corresponding to a geodesic because $\tilde{\phi}(s, t)$ is not necessarily a geodesic, i.e. $V_{G}\left(\xi_{x \nu \omega}(t), \hat{\xi}_{\hat{x} \hat{\nu} \hat{\omega}}(t)\right) \leq l(t)$, one has:

$$
\begin{aligned}
V_{G}\left(\xi_{x \nu \omega}(t), \hat{\xi}_{\hat{x} \hat{\nu} \hat{\omega}}(t)\right) & \leq \mathrm{e}^{-\lambda t} V_{G}\left(\xi_{x \nu \omega}(0), \hat{\xi}_{\hat{x} \hat{\nu} \hat{\omega}}(0)\right) \\
+\frac{1}{\lambda} \psi_{\text {ext }}\left(\|\hat{\nu}\|_{\infty}\right) & +\frac{1}{\lambda} \psi_{\text {int }}\left(\|\omega-\hat{\omega}\|_{\infty}\right)
\end{aligned}
$$

For every $x \in \mathbb{R}^{n}, \hat{x} \in \mathbb{R}^{\hat{n}}$, we use (III.1) and the Schwarz inequality to obtain:

$$
\begin{aligned}
& \alpha\left\|h_{1}(x)-\hat{h}_{1}(\hat{x})\right\|^{2}=\alpha\|\tilde{h}(\tilde{x})\|^{2} \\
& \leq \alpha\left(\int_{0}^{1} \sqrt{\chi^{\prime}(s)^{T} \frac{\partial \tilde{h}_{1}}{\partial \tilde{x}}(\chi(s))^{T} \frac{\partial \tilde{h}_{1}}{\partial \tilde{x}}(\chi(s)) \chi^{\prime}(s)} d s\right)^{2} \\
& \leq\left(\int_{0}^{1} \sqrt{\chi^{\prime}(s)^{T} G(\chi(s)) \chi^{\prime}(s)} d s\right)^{2} \\
& \leq \int_{0}^{1} \chi^{\prime}(s)^{T} G(\chi(s)) \chi^{\prime}(s) d s=V_{G}(\tilde{x})
\end{aligned}
$$

where $\tilde{x}=[x ; \hat{x}]$. Combining (III.5) with (III.4), one can conclude that (III.3) is satisfied with $\beta(r, s)=$ $\sqrt{\frac{r}{\alpha}} \mathrm{e}^{-\frac{\lambda}{2} s}, \quad \bar{\psi}_{\text {ext }}(r)=\sqrt{\frac{1}{\alpha \lambda} \psi_{\text {ext }}(r)}$, and $\bar{\psi}_{\text {int }}(r)=$ $\sqrt{\frac{1}{\alpha \lambda} \psi_{\text {int }}(r)}, \forall s, r \in \mathbb{R}_{\geq 0}$.

\section{INTERCONNECTED SYSTEMS}

Here, we define the interconnection between the control subsystems by defining the relationship between the outputs and internal inputs. Consider $N \in \mathbb{N}_{\geq 1}$ control subsystems

$$
\Sigma_{i}=\left(\mathcal{M}_{n_{i}}, \mathbb{R}^{m_{i}}, \mathbb{R}^{p_{i}}, \mathcal{U}_{i}, \mathcal{W}_{i}, f_{i}, \mathbb{R}^{q_{i}}, h_{i}\right),
$$

$i \in[1 ; N]$, with partitioned internal inputs and outputs

$$
\begin{aligned}
w_{i} & =\left[w_{i 1} ; \ldots ; w_{i(i-1)} ; w_{i(i+1)} ; \ldots ; w_{i N}\right], \quad w_{i j} \in \mathbb{R}^{p_{i j}} \\
y_{i} & =\left[y_{i 1} ; \ldots ; y_{i N}\right], \quad y_{i j} \in \mathbb{R}^{q_{i j}}
\end{aligned}
$$

and the output function

$$
h_{i}\left(x_{i}\right)=\left[h_{i 1}\left(x_{i}\right) ; \ldots ; h_{i N}\left(x_{i}\right)\right] .
$$

We interpret the outputs $y_{i i}$ as external ones, whereas the outputs $y_{i j}$ with $i \neq j$ are internal ones which are used to define the interconnected control system. In particular, we assume that the dimension of $w_{i j}$ is equal to the dimension of $y_{j i}$ i.e. the following dimension constraints hold:

$$
p_{i j}=q_{j i}, \quad \forall i, j \in[1 ; N], i \neq j .
$$

If there is no connection from the control system $\Sigma_{i}$ to $\Sigma_{j}$, then we assume that the connecting output function is identically zero for all arguments i.e. $h_{i j} \equiv 0$. Now we provide the definition of the interconnected control system.

Definition 4.1: Consider $N \in \mathbb{N}_{\geq 1}$ control subsystems

$$
\Sigma_{i}=\left(\mathcal{M}_{n_{i}}, \mathbb{R}^{m_{i}}, \mathbb{R}^{p_{i}}, \mathcal{U}_{i}, \mathcal{W}_{i}, f_{i}, \mathbb{R}^{q_{i}}, h_{i}\right),
$$

$i \in[1 ; N]$, with the input-output configuration given by (IV.1), (IV.2) and (IV.3). The interconnected control system

$$
\Sigma=\left(\mathcal{M}_{n}, \mathbb{R}^{m}, \mathcal{U}, f, \mathbb{R}^{q}, h\right),
$$

denoted by $\mathcal{I}\left(\Sigma_{1}, \ldots, \Sigma_{N}\right)$, follows by $\mathcal{M}_{n}=\Pi_{i=1}^{N} \mathcal{M}_{n_{i}}$, $m=\sum_{i=1}^{N} m_{i}, q=\sum_{i=1}^{N} q_{i i}$ and the functions

$$
\begin{aligned}
f(x, u) & =\left[f_{1}\left(x_{1}, u_{1}, w_{1}\right) ; \ldots ; f_{N}\left(x_{N}, u_{N}, w_{N}\right)\right], \\
h(x) & =\left[h_{11}(x) ; \ldots ; h_{N N}\left(x_{N}\right)\right],
\end{aligned}
$$

where $u=\left[u_{1} ; \ldots ; u_{N}\right], x=\left[x_{1} ; \ldots ; x_{N}\right]$, and the interconnection variables constrained by $w_{i j}=y_{j i}$, for all $i, j \in[1 ; N], i \neq j$. 


\section{Compositionality Result}

In this section we provide sufficient conditions under which an interconnection of abstractions of control systems, is an abstraction of the original interconnected system. We assume that we are given $N \in \mathbb{N}$ control systems

$$
\Sigma_{i}=\left(\mathcal{M}_{n_{i}}, \mathbb{R}^{m_{i}}, \mathbb{R}^{p_{i}}, \mathcal{U}_{i}, \mathcal{W}_{i}, f_{i}, \mathbb{R}^{q_{i}}, h_{i}\right),
$$

where $i \in[1 ; N]$, together with the corresponding abstractions

$$
\hat{\Sigma}_{i}=\left(\mathcal{M}_{\hat{n}_{i}}, \mathbb{R}^{\hat{m}_{i}}, \mathbb{R}^{p_{i}}, \hat{\mathcal{U}}_{i}, \hat{\mathcal{W}}_{i}, \hat{f}_{i}, \mathbb{R}^{q_{i}}, \hat{h}_{i}\right),
$$

where $i \in[1 ; N]$ and with simulation function $V_{G_{i}}$, associated with the (pseudo) Riemannian structure $G_{i}$, from $\hat{\Sigma}_{i}$ to $\Sigma_{i}$. We use $\alpha_{i}, \lambda_{i}, \psi_{i \text { ext }}$, and $\psi_{i \text { int }}$ to denote the corresponding constants and functions appearing in Definition 3.1. We require the following assumptions in order to provide the compositionality result:

Assumption 1: For any $i, j \in[1 ; N], i \neq j$, there exists a positive constant $\delta_{i j}$ such that for any $s \in \mathbb{R}_{\geq 0}$ :

$$
\begin{aligned}
& h_{j i} \equiv 0 \Longrightarrow \delta_{i j}=0 \text { and } \\
& h_{j i} \neq 0 \Longrightarrow \psi_{i \text { int }}\left((N-1) \sqrt{\frac{s}{\alpha_{j}}}\right) \leq \delta_{i j} s .
\end{aligned}
$$

For notational simplicity we define the matrix $\Delta \in \mathbb{R}^{N \times N}$ with its components given by $\Delta_{i i}=0$ for $i \in[1 ; N]$ and $\Delta_{i j}=\delta_{i j}$ for $i, j \in[1 ; N], i \neq j$. The next theorem provides a compositionality approach on the construction of abstractions of interconnected control systems and that of the corresponding simulation functions.

Theorem 5.1: Consider the interconnected control system $\Sigma=\mathcal{I}\left(\Sigma_{1}, \ldots, \Sigma_{N}\right)$, induced by $N$ control subsystems $\Sigma_{i}$. Suppose each subsystem $\Sigma_{i}$ admits an abstraction $\hat{\Sigma}_{i}$ with the corresponding simulation function $V_{G_{i}}$ with respect to (pseudo) Riemannian metric $G_{i}$. If Assumption 1 holds and there exists a vector $\mu=\left[\mu_{1} ; \ldots ; \mu_{N}\right]$, where $\mu_{i} \geq 1 \forall i \in$ $[1 ; N]$, such that the inequality

$$
\mu^{T}(-\Lambda+\Delta)<0
$$

is satisfied ${ }^{3}$, where $\Lambda=\operatorname{diag}\left\{\lambda_{1}, \ldots, \lambda_{N}\right\}$, then

$$
V_{G}(\tilde{x})=\inf _{\tilde{\gamma} \in \Gamma(\tilde{x}, 0)} \int_{0}^{1} \tilde{\gamma}^{\prime}(s)^{T} G(\tilde{\gamma}(s)) \tilde{\gamma}^{\prime}(s) d s,
$$

is a simulation function from the interconnected control system $\hat{\Sigma}=\mathcal{I}\left(\hat{\Sigma}_{1}, \ldots, \hat{\Sigma}_{N}\right)$ to $\Sigma$, where

$$
G(\tilde{x})=\left[\begin{array}{cccc}
\mu_{1} G_{1}\left(\tilde{x}_{1}\right) & 0 & \cdots & 0 \\
0 & \mu_{2} G_{2}\left(\tilde{x}_{2}\right) & & 0 \\
\vdots & & \ddots & \vdots \\
0 & \ldots & 0 & \mu_{N} G_{N}\left(\tilde{x}_{N}\right)
\end{array}\right],
$$

$\tilde{x}=\left[\tilde{x}_{1} ; \ldots ; \tilde{x}_{N}\right]$, and $\tilde{x}_{i}=\left[x_{i} ; \hat{x}_{i}\right] \in \mathcal{M}_{n_{i}} \times \mathcal{M}_{\hat{n}_{i}} \forall i \in$ $[1 ; N]$.

${ }^{3}$ We interpret the inequality component-wise i.e. for $x \in \mathbb{R}^{N}$ we have $x<0$ iff every entry $x_{i}<0, i \in\{1, \ldots, N\}$
Proof: For any $x_{i} \in \mathcal{M}_{n_{i}}$, and $\hat{x}_{i} \in \mathcal{M}_{\hat{n}_{i}}, i \in[1 ; N]$, define

$$
\tilde{h}(\tilde{x}):=\left[\begin{array}{c}
\tilde{h}_{1}\left(\tilde{x}_{1}\right) \\
\vdots \\
\tilde{h}_{N}\left(\tilde{x}_{N}\right)
\end{array}\right]:=\left[\begin{array}{c}
h_{1}\left(x_{1}\right)-\hat{h}_{1}\left(\hat{x}_{1}\right) \\
\vdots \\
h_{N}\left(x_{N}\right)-\hat{h}_{N}\left(\hat{x}_{N}\right)
\end{array}\right],
$$

where $\tilde{x}=\left[\tilde{x}_{1} ; \ldots ; \tilde{x}_{N}\right]$, and $\tilde{x}_{i}=\left[x_{i} ; \hat{x}_{i}\right], \forall i \in[1 ; N]$. One has:

$$
\frac{\partial \tilde{h}}{\partial \tilde{x}}(\tilde{x})=\left[\begin{array}{cccc}
\frac{\partial \tilde{h}_{1}}{\partial \tilde{x}_{1}}\left(\tilde{x}_{1}\right) & 0 & \ldots & 0 \\
0 & \frac{\partial \tilde{h}_{2}}{\partial \tilde{x}_{2}}\left(\tilde{x}_{2}\right) & \ldots & 0 \\
\vdots & & \ddots & \vdots \\
0 & \ldots & 0 & \frac{\partial \tilde{h}_{N}}{\partial \tilde{x}_{N}}\left(\tilde{x}_{N}\right)
\end{array}\right]
$$

and

$$
\begin{aligned}
& \underline{\alpha}\left(\frac{\partial \tilde{h}}{\partial \tilde{x}}\right)^{T} \frac{\partial \tilde{h}}{\partial \tilde{x}} \\
& \preceq \operatorname{diag}\left\{\alpha_{1}\left(\frac{\partial \tilde{h}_{1}}{\partial \tilde{x}_{1}}\right)^{T} \frac{\partial \tilde{h}_{1}}{\partial \tilde{x}_{1}}, \ldots, \alpha_{N}\left(\frac{\partial \tilde{h}_{N}}{\partial \tilde{x}_{N}}\right)^{T} \frac{\partial \tilde{h}_{N}}{\partial \tilde{x}_{N}}\right\} \\
& \preceq\left[\begin{array}{cccc}
\mu_{1} G_{1}\left(\tilde{x}_{1}\right) & 0 & \ldots & 0 \\
0 & \mu_{2} G_{2}\left(\tilde{x}_{2}\right) & & 0 \\
\vdots & & \ddots & \vdots \\
0 & \ldots & 0 & \mu_{N} G_{N}\left(\tilde{x}_{N}\right)
\end{array}\right]=G(\tilde{x}),
\end{aligned}
$$

where $\underline{\alpha}=\min \left\{\alpha_{1}, \ldots, \alpha_{N}\right\}$. Thus, the condition (III.1) is satisfied with $\alpha=\underline{\alpha}$. Now we prove inequality (III.2). For all $i \in[1 ; N]$, consider any $\tilde{x}_{i}=\left[x_{i} ; \hat{x}_{i}\right] \in \mathcal{M}_{n_{i}} \times \mathcal{M}_{\hat{n}_{i}}$, $\delta x_{i}=\left[\delta x_{i} ; \delta \hat{x}_{i}\right] \in \mathcal{T}_{x_{i}} \mathcal{M}_{n_{i}} \times \mathcal{T}_{\hat{x}_{i}} \mathcal{M}_{\hat{n}_{i}}, \hat{u}_{i} \in \mathbb{R}^{\hat{m}_{i}}$, and any $\delta \hat{u}_{i} \in \mathbb{R}^{\hat{m}_{i}}$. Under the map $u_{i}=k_{i}\left(\tilde{x}_{i}, \hat{u}_{i}, \hat{w}_{i}\right)$, (III.2) is satisfied for each pair of subsystems $\Sigma_{i}$ and $\hat{\Sigma}_{i}$, with the internal inputs given by $w_{i j}=y_{j i}=h_{j i}\left(x_{j}\right)$, and $\hat{w}_{i j}=\hat{y}_{j i}=\hat{h}_{j i}\left(\hat{x}_{j}\right)$. The corresponding differential internal inputs are given $\delta w_{i j}=\delta y_{j i}=\frac{\partial h_{j i}}{\partial x_{j}} \delta x_{j}$, and $\delta \hat{w}_{i j}=\delta \hat{y}_{j i}=$ $\frac{\partial \hat{h}_{j i}}{\partial \hat{x}_{j}} \delta \hat{x}_{j}$. We consider the time derivative of the function $\mathcal{S}(\tilde{x}, \delta \tilde{x})=\delta \tilde{x}^{T} G(\tilde{x}) \delta \tilde{x}$ along the solution trajectory and employ the conditions (V.1) which results in the chain of inequalities (V.2), where we use the triangle inequality and the following inequality [11]

$$
\psi_{i \mathrm{int}}\left(r_{1}+\cdots+r_{N-1}\right) \leq \sum_{i=1}^{N-1} \psi_{i \mathrm{int}}\left((N-1) r_{i}\right) .
$$

We define the vector $\delta \hat{u}=\left[\delta \hat{u}_{1} ; \ldots ; \delta \hat{u}_{N}\right]$, and the function

$$
\psi_{\text {ext }}(s):=\left\{\begin{array}{ll}
\max _{\vec{s} \geq 0} & \sum_{i=1}^{N} \mu_{i} \psi_{i \text { ext }}\left(s_{i}\right) \\
\text { s.t. } & \|\vec{s}\|=s .
\end{array},\right.
$$

where $\vec{s}=\left[s_{1} ; \ldots ; s_{N}\right] \in \mathbb{R}^{N}, \psi_{\text {ext }} \in \mathcal{K}_{\infty} \cup\{0\}$. Therefore, one has:

$$
\dot{S} \leq-\lambda \delta \tilde{x}^{T} G(\tilde{x}) \delta \tilde{x}+\psi_{\text {ext }}(\|\delta \hat{u}\|),
$$

where $\lambda$ is the minimum element of the vector

$$
-\mu^{T}(-\Lambda+\Delta)
$$




$$
\begin{aligned}
& \dot{S}=\frac{d}{d t} \mu_{i} \sum_{i=1}^{N} \delta \tilde{x}_{i}^{T} G_{i}\left(\tilde{x}_{i}\right) \delta \tilde{x}_{i} \leq \sum_{i=1}^{N} \mu_{i}\left(-\lambda_{i} \delta \tilde{x}_{i}^{T} G_{i}\left(\tilde{x}_{i}\right) \delta \tilde{x}_{i}+\psi_{i \operatorname{ext}}\left(\left\|\delta \hat{u}_{i}\right\|\right)+\psi_{i \mathrm{int}}\left(\left\|\delta w_{i}-\delta \hat{w}_{i}\right\|\right)\right) \\
& \leq \sum_{i=1}^{N} \mu_{i}\left(-\lambda_{i} \delta \tilde{x}_{i}^{T} G_{i}\left(\tilde{x}_{i}\right) \delta \tilde{x}_{i}+\sum_{j=1, j \neq i}^{N} \psi_{i \mathrm{int}}\left((N-1)\left\|\delta w_{i j}-\delta \hat{w}_{i j}\right\|\right)\right)+\sum_{i=1}^{N} \mu_{i} \psi_{i \operatorname{ext}}\left(\left\|\delta \hat{u}_{i}\right\|\right) \\
& =\sum_{i=1}^{N} \mu_{i}\left(-\lambda_{i} \delta \tilde{x}_{i}^{T} G_{i}\left(\tilde{x}_{i}\right) \delta \tilde{x}_{i}+\sum_{j=1, j \neq i}^{N} \psi_{i \mathrm{int}}\left((N-1)\left\|\delta y_{j i}-\delta \hat{y}_{j i}\right\|\right)\right)+\sum_{i=1}^{N} \mu_{i} \psi_{\text {iext }}\left(\left\|\delta \hat{u}_{i}\right\|\right) \\
& =\sum_{i=1}^{N} \mu_{i}\left(-\lambda_{i} \delta \tilde{x}_{i}^{T} G_{i}\left(\tilde{x}_{i}\right) \delta \tilde{x}_{i}+\sum_{j=1, j \neq i}^{N} \psi_{i \mathrm{int}}\left((N-1)\left\|\frac{\partial h_{j}}{\partial x_{j}} \delta x_{j}-\frac{\partial \hat{h}_{j}}{\partial \hat{x}_{j}} \delta \hat{x}_{j}\right\|\right)\right)+\sum_{i=1}^{N} \mu_{i} \psi_{i \mathrm{ext}}\left(\left\|\delta \hat{u}_{i}\right\|\right) \\
& =\sum_{i=1}^{N} \mu_{i}\left(-\lambda_{i} \delta \tilde{x}_{i}^{T} G_{i}\left(\tilde{x}_{i}\right) \delta \tilde{x}_{i}+\sum_{j=1, j \neq i}^{N} \psi_{i \mathrm{int}}\left((N-1)\left\|\frac{\partial \tilde{h}_{j}}{\partial \tilde{x}_{j}} \delta \tilde{x}_{j}\right\|\right)\right)+\sum_{i=1}^{N} \mu_{i} \psi_{i \operatorname{ext}}\left(\left\|\delta \hat{u}_{i}\right\|\right) \\
& \leq \sum_{i=1}^{N} \mu_{i}\left(-\lambda_{i} \delta \tilde{x}_{i}^{T} G_{i}\left(\tilde{x}_{i}\right) \delta \tilde{x}_{i}+\sum_{j=1, j \neq i}^{N} \psi_{i \mathrm{int}}\left(\frac{1}{\sqrt{\alpha_{j}}}(N-1) \sqrt{\delta \tilde{x}_{j}^{T} G_{j}\left(\tilde{x}_{j}\right) \delta \tilde{x}_{j}}\right)\right)+\sum_{i=1}^{N} \mu_{i} \psi_{i \operatorname{ext}}\left(\left\|\delta \hat{u}_{i}\right\|\right) \\
& \leq \sum_{i=1}^{N} \mu_{i}\left(-\lambda_{i} \delta \tilde{x}_{i}^{T} G_{i}\left(\tilde{x}_{i}\right) \delta \tilde{x}_{i}+\sum_{j=1, j \neq i}^{N} \delta_{i j} \delta \tilde{x}_{j}^{T} G_{j}\left(\tilde{x}_{j}\right) \delta \tilde{x}_{j}\right)+\sum_{i=1}^{N} \mu_{i} \psi_{i \mathrm{ext}}\left(\left\|\delta \hat{u}_{i}\right\|\right) \\
& =\mu^{T}(-\Lambda+\Delta)\left[\delta \tilde{x}_{1}^{T} G\left(\tilde{x}_{1}\right) \delta \tilde{x}_{1} ; \ldots ; \delta \tilde{x}_{N}^{T} G\left(\tilde{x}_{N}\right) \delta \tilde{x}_{N}\right]+\sum_{i=1}^{N} \mu_{i} \psi_{i \operatorname{ext}}\left(\left\|\delta \hat{u}_{i}\right\|\right)
\end{aligned}
$$

which satisfies inequality (III.2) with $\psi_{\text {int }} \equiv 0$. Hence we conclude that

$$
V(x, \hat{x})=\inf _{\tilde{\gamma} \in \Gamma(\tilde{x}, 0)} \int_{0}^{1} \tilde{\gamma}^{\prime}(s)^{T} G(\tilde{\gamma}(s)) \tilde{\gamma}^{\prime}(s) d s,
$$

is a simulation function from $\hat{\Sigma}$ to $\Sigma$.

\section{CONCLUSION}

In this work, using tools from differential geometry, we derived sufficient conditions based on small-gain type reasoning under which abstractions of interconnected systems evolving on smooth Riemannian manifolds can be constructed compositionally. In future work, we will look at deriving constructive conditions which facilitate the construction of abstractions for various classes of nonlinear systems evolving on smooth Riemannian manifolds together with the corresponding simulation functions and interface maps, hence, generalizing the results in [12], which is only applicable to linear systems over Euclidean spaces.

\section{REFERENCES}

[1] G. Pola, P. Pepe, and M. D. Di Benedetto, "Symbolic models for networks of control systems," IEEE Transactions on Automatic Control, vol. 61, no. 11, pp. 3663-3668, November 2016.

[2] Y. Tazaki and J.-i. Imura, "Bisimilar finite abstractions of interconnected systems," in Proceedings of the 11th International Conference on Hybrid Systems: Computation and Control, April 2008, pp. 514527.

[3] M. Rungger and M. Zamani, "Compositional construction of approximate abstractions," in Proceedings of the 18th International Conference on Hybrid Systems: Computation and Control, April 2015, pp. $68-77$.
[4] M. Zamani, M. Rungger, and P. M. Esfahani, "Approximations of stochastic hybrid systems: A compositional approach," IEEE Transactions on Automatic Control, vol. 62, no. 6, pp. 2838-2853, 2017.

[5] A. U. Awan and M. Zamani, "Compositional abstractions of networks of stochastic hybrid systems: A dissipativity approach," IFAC-PapersOnLine, vol. 50, no. 1, pp. 15804 15809, 2017, 20th IFAC World Congress. [Online]. Available: http://www.sciencedirect.com/science/article/pii/S2405896317331361

[6] F. Bullo and A. D. Lewis, Geometric control of mechanical systems: modeling, analysis, and design for simple mechanical control systems. Springer Science \& Business Media, 2004, vol. 49.

[7] F. Taringoo, D. Nei, Y. Tan, and P. M. Dower, "Averaging for nonlinear systems on riemannian manifolds," in 52nd IEEE Conference on Decision and Control, Dec 2013, pp. 654-659.

[8] S. Dashkovskiy, B. S. Rüffer, and F. R. Wirth, "An ISS small gain theorem for general networks," Mathematics of Control, Signals, and Systems, vol. 19, no. 2, pp. 93-122, 2007.

[9] F. Forni and R. Sepulchre, "A differential Lyapunov framework for contraction analysis," IEEE Transactions on Automatic Control, vol. 59, no. 3, pp. 614-628, March 2014.

[10] H. K. Khalil, "Nonlinear systems," Prentice-Hall, New Jersey, vol. 2, no. 5 , pp. 5-1, 1996.

[11] C. M. Kellett, "A compendium of comparison function results," Mathematics of Control, Signals, and Systems, vol. 26, no. 3, pp. 339374, 2014.

[12] M. Rungger and M. Zamani, "Compositional construction of approximate abstractions of interconnected control systems," IEEE Transactions on Control of Network Systems, vol. 5, no. 1, pp. 116-127, March 2018. 